\title{
Vocación docente de la educación escolar básica, a partir de las variables extrínsecas e intrínsecas
}

\author{
Miryam Rossana Salinas Rojas \\ miryam.salinas@gmail.com \\ Maestría en Ciencias de la Educación, \\ con énfasis en Investigación Científica \\ Facultad de Humanidades y Ciencias de la Educación \\ Universidad Nacional de Pilar
}

\section{RESUMEN}

La investigación tiene como objetivo analizar la vocación docente de la "Esc. Bás. $\mathrm{N}^{\mathrm{o}}$ 5639 Ntra del Pilar" y la "Esc. Bás. N 1.142 Santa María Magdalena” de la Ciudad de Pilar, a partir de las variables extrínsecas e intrínsecas, siendo que la rápida evolución de la sociedad moderna exige de la educación actual posturas que le lleven a encarar las situaciones que configuran los actores que inciden en ella; es decir, involucrar a docentes, padres y alumnos. Para la recolección de los datos e información se utiliza el cuestionario, esta técnica se aplicó a los docentes de las escuelas seleccionadas, la entrevista, dirigido a los directivos de las instituciones. Se ha llegado a la conclusión que, desde un punto de vista organizativo, la educación tiene como objetivo transmitir conocimientos y creencias, esto permite captar el sistema educativo. Las condiciones laborales son retos inexorables para cualquier institución, pues existen evidencias suficientes que permiten afirmar que las buenas condiciones de trabajo son fundamentales para que todo trabajador rinda en forma a la hora de su desempeño. La actividad del docente en el aula es determinante para la enseñanza que éste desarrolla en frutos de aprendizaje en los alumnos. Y para que esto ocurra, los docentes están poniendo todos sus empeños de manera a propiciar las condiciones mínimas favorables en el proceso de enseñanza-aprendizaje.

Palabras claves: vocación docente; variables extrínsecas, variables intrínsecas 


\title{
Teaching vocation of basic school education, from the extrinsic and intrinsic variables
}

\begin{abstract}
The objective of the research is to analyze the teaching vocation of the "Esc. Bas. $\mathrm{N}^{\mathrm{o}}$ 5639 Ntra del Pilar" and "Esc. Bas. N 1,142 Santa María Magdalena" of the City of Pilar, based on the extrinsic and intrinsic variables, since the rapid evolution of modern society demands from current education positions that lead it to face the situations that configure the actors that affect her; that is, involve teachers, parents and students. For the collection of data and information, the questionnaire is used, this technique was applied to the teachers of the selected schools, the interview, directed to the directors of the institutions. It has been concluded that, from an organizational point of view, education aims to transmit knowledge and beliefs, this allows to capture the educational system. Working conditions are inexorable challenges for any institution, as there is sufficient evidence to affirm that good working conditions are essential for every worker to perform in good shape when it comes to their performance. The activity of the teacher in the classroom is decisive for the teaching that it develops in the fruits of learning in the students. And for this to happen, teachers are putting all their efforts in order to promote the minimum favorable conditions in the teaching-learning process.
\end{abstract}

Keywords: teaching vocation; extrinsic variables, intrinsic variables.

Artículo recibido: 15 octubre. 2021 Aceptado para publicación: 18 noviembre 2021 Correspondencia: miryam.salinas@gmail.com

Conflictos de Interés: Ninguna que declarar 


\section{INTRODUCCIÓN}

La mayor incorporación de docentes a la vida socio-productiva no ha sido acompañada por sistemas de corresponsabilidad social y familiar en el trabajo del hogar y los cuidados. Todo esto da lugar a fuertes conflictos entre la vida personal, familiar y laboral de los trabajadores, y las lleva en muchos casos a discontinuar carreras profesionales e iniciativas productivas.

Con este trabajo se intenta aprehender un área de la cuestión social de nuestra comunidad, escasamente investigado y analizado, el abordaje de esta problemática que posee un amplio nivel de pertinencia como profesionales de la educación al analizar la vocación docente de la "Esc. Bás. No 5639 Ntra del Pilar" y la "Esc. Bás. No 1.142 Santa María Magdalena de la Ciudad de Pilar, año 2019-2020, a partir de las variables extrínsecas e intrínsecas.

La idea educativa puede interpretarse como el medio de transmitir el conocimiento y de formar a las personas en los valores de la sociedad, concebida como sinónimo de socialización, es un tipo de relación interpersonal que acontece en un marco social.

Durante la mayor parte de la historia humana la instrucción deliberada ha sido algo esporádico e incidental, y aun en las sociedades más complejas gran parte de la instrucción, en el sentido más amplio del término, se imparte a través de organismos que son las escuelas.

La vocación docente es un contenido del currículo oculto, ya que, al momento de decidir la profesión de pedagogía, este componente construido desde la subjetividad no participa formalmente del proceso de la toma de decisión; no obstante, esta situación no le resta importancia, pues el deseo por el cual una persona decide ser educador tiene un importante significado para el futuro profesional docente (Mujica y Orellana, 2019).

De acuerdo a Martín, Martín, Dapelo, Pizarro, Gusto \& otros, (2015). La vocación también tiene dimensiones tales como las características de la personalidad eficaz, como son la autoestima, asertividad, capacidad de trabajo, confianza en sí mismo, estabilidad emocional, estilo de pensamiento, extraversión, flexibilidad, independencia, iniciativa, locus de control, motivación de logro, optimismo, perseverancia, tolerancia a la incertidumbre, toma de riesgos y valores personales.

La educación en todo el mundo es un proceso complejo que ha sido dirigido por el ser humano para múltiples fines, los cuales se han generado a partir de las diferentes 
necesidades sociales según cada sitio geográfico. A pesar de ser un proceso tan amplio en su cobertura territorial y de áreas de estudio, ha generado elementos en común que pueden ser apreciados en las diferentes partes del mundo, como las instituciones educativas que van progresando según el nivel etario y que culminan la formación en la Universidad o Instituto, ya sea de carácter técnico o profesional (Mujica y Orellana, 2019).

La importancia de este trabajo consiste en que permite reflexionar acerca de la vocación docente a partir de las variables extrínsecas e intrínsecas, cuyos resultados redundarán para la implementación de políticas institucionales, departamentales y/o nacionales, si se quiere para mejorar las condiciones laborales de los mismos, cuya intervención es clave para el desarrollo de este país.

En el ámbito educativo, estos estudios son escasos, y poco importantes, ya que por décadas se ha considerado que abrazar la docencia era una fuente laboral inmediata.

El estudio es viable, considerando que existe la predisposición por parte de las autoridades, los docentes, es de interés de la comunidad educativa toda, analizar la situación del contexto real donde se desarrolla la interacción docente-alumno y su clima.

La importancia de esta investigación radica en que aporta informaciones actuales y veraces que servirán a todas las personas del estamento educativo como también para futuros proyectos de otras investigaciones.

\section{MATERIALES Y MÉTODOS}

A continuación, se presentan antecedentes de estudios que podrán adecuarse al sistema metodológico a utilizar.

Fernández, González, Pompa Garcés, Figueredo, (2016) y Ávila, (2016), realizaron un estudio descriptivo prospectivos seleccionado en ambos casos una muestra considerable de estudiantes para analizar las motivaciones de elección de la carrera, luego estas muestras fueron analizadas y los resultados proyectados a nivel poblacional. Es así como se desea desarrollar el presente trabajo, utilizando muestras de estudiantes y docentes para luego estimar los porcentajes poblacionales. Esta investigación es cualitativa, así como (Gómez, Flores, Giménez, 1996) y (Hernández, 2005) detallan que la metodología de la investigación cualitativa, en resultados, se analizan de manera 
cualitativa, categorizándose las respuestas en función de los indicadores de las dimensiones estudiadas.

La población de estudio constituye los docentes de la Esc. Bás. № 5639 Ntra del Pilar” y la “Esc. Bás. No 1.142 Santa María Magdalena, de la Ciudad de Pilar, año 2019-2020. Se elegiron todos los docentes de ambos turnos de las instituciones educativas seleccionadas.

Los datos primarios son obtenidos de fuentes bibliográficas varias, encontradas con el buscador de Google académico. Entre dichas fuentes se encuentran revistas con publicación de revisiones bibliográficas, artículos científicos o trabajos de grado.

Los datos secundarios son recabados de los informantes, en este caso de docentes de las instituciones en estudio.

Para la selección de información se priorizaron las informaciones actualizadas, en caso de algunos libros de autores conocidos en el área son utilizados a pesar de la antigüedad puesto que sus definiciones aún son válidas. Se eligen materiales publicados en revistas de reconocida trayectoria.

Las encuestas son contestadas por los docentes en total libertad y privacidad, a modo de no influir en las respuestas, son contabilizadas y representadas en gráfico para mejor apreciación de los resultados.

Miranda (2010), menciona que en la investigación descriptiva se realiza en el ambiente natural donde se encuentran los fenómenos estudiados. Los datos recogidos pueden ser cualitativos o cuantitativos. La investigación fenomenológica trata de describir y comprender las situaciones y los procesos de manera integral y profunda, considerando inclusive el contexto que le rodea a la problemática estudiada.

La investigación es de método cualitativo y cuantitativo, cualitativo para la descripción de las variables estudiadas y cuantitativas para la cuantificación previa a los análisis matemáticos de las mismas.

Según Bernal (2006) un aspecto de alta relevancia dentro del proceso de investigación es la obtención de la información, ya que de esta depende tanto la confiabilidad como la validez del estudio (p.15).

Así mismo, Bernal (2006, p. 163) señala que dentro del proceso de investigación científica existe una gran variedad de técnicas e instrumentos para la colecta de 
información y que el tipo de instrumento o técnica a utilizar varía respecto al tipo de investigación que se va a realizar sea esta cualitativa o cuantitativa.

Los resultados obtenidos de las diferentes técnicas de recolección de datos se analizaron en dos momentos; Primeramente; se analizaron los datos cuantitativos, donde se tabularon los datos y se cargaron sobre una matriz Excel 2010, sobre esta matriz se graficaron y se representaron los datos en forma porcentual, mediante gráficos de barras.

Entrevista: Es una conversación dirigida, con un propósito específico y que usa un formato de preguntas y respuestas. Una entrevista es un dialogo en el que la persona (entrevistador), hace una serie de preguntas a otra persona (entrevistado), con el fin de conocer mejor sus ideas, sus sentimientos su forma de actuar, la misma fue aplicada a los directores.

Cuestionario: Es un conjunto de preguntas normalizadas dirigidas a una muestra representativa de la población o instituciones, con el fin de conocer estados de opinión o hechos específicos. La intención de la encuesta no es describir los individuos particulares quienes, por azar, son parte de la muestra sino obtener un perfil compuesto de la población. Una "encuesta" recoge información de una "muestra." Una "muestra" es usualmente sólo una porción de la población bajo estudio, este instrumento de recolección de datos fue aplicado a los Docentes.

Una vez diseñado el instrumento, fueron sometido a dos procesos antes de su aplicación: la validez y la confiabilidad. En tal contexto, Hernández y otros (2008), indican que, "La validez, la confiabilidad y la objetividad no deben tratarse de forma separada" (p.288)

Para llevar a cabo este proceso, se construyó un formato de validación, el cual incluye las instrucciones sobre las cuales deben guiarse los jueces expertos y emitir su opinión, para realizarle las modificaciones pertinentes, garantizando que el instrumento mida los aspectos de estudio, al respecto Hernández y otros (2008), indican que "se refiere al grado en que un instrumento realmente mide la variable que pretende medir" (p.277).

Es decir, se relacionó la coherencia entre el instrumento y lo que se desea medir. En cuanto a la confiabilidad, Hernández y otros (2008) dicen que "se refiere al grado en que su aplicaron repetida al mismo sujeto u objeto produce resultados iguales (p.277). 


\section{RESULTADO Y DISCUSIONES}

Figura 1. Factores extrínsecos -Factores intrínsecos- Clima laboral desarrollado

\section{Factores extrínsecos -Factores intrínsecos- Clima laboral desarrollado}

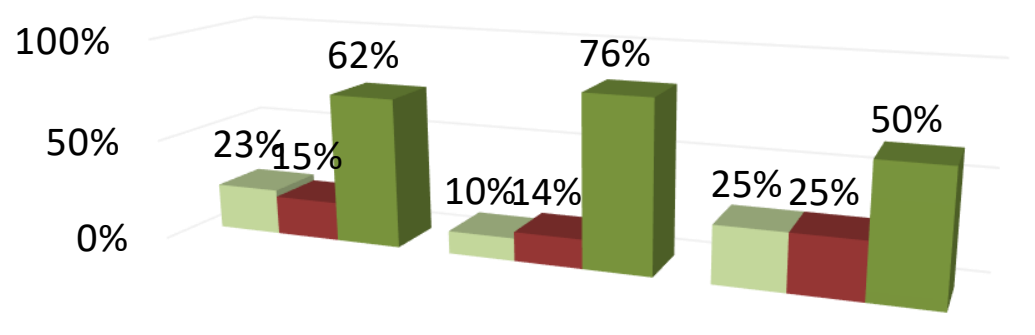

FACTORES EXTRÍNSECOS - FACTORES INTRÍNSECOS- CLIMA LABORAL DESARROLLADO
(1) Nunca
(2) A veces
(3) Siempre

Análisis pedagógico: Luego del análisis estadístico procesado en porcentaje la variable de investigación factores extrínsecos donde las subdimensiones: remuneración, burocracias impuestas, la presión de los padres, como amenazas que pongan en riesgo la labor docente, se ha obtenido un nivel elevado $62 \%$ que alcanza la escala siempre, seguido de la opción nunca con el $23 \%$ y por último a veces con $15 \%$, datos que indican un elevado nivel de factores extrínsecas que aparecen como amenazas que ponen en riesgo la labor docente.

La enseñanza se encuentra actualmente ante muchas vicisitudes que enturbian una fascinante labor, como es la docencia. Según Cárdenas y Santos (2016). La sincronía en la docencia se entiende como el conjunto de aspectos simultáneos que impactan la vida cotidiana de los profesores, tanto en el entorno mundial, nacional y local como en el interior de la escuela: desde las tendencias educativas del mundo en el siglo XXI y la legislación nacional profesional hasta las tareas académicas, educativas y administrativas que realiza en el centro escolar, pasando por la relación con el personal directivo y administrativo. La dimensión jurídico-política norma el ejercicio profesional y lo determina. La tarea académica incluye los momentos de planeación, dinámica didáctica y evaluación. La tarea educativa consiste en la formación de los alumnos en aspectos éticos y axiológicos. Las funciones administrativas, a las que suelen resistirse los maestros, incluyen los formatos de registro y control escolar. Finalmente, los profesores mantienen relaciones sincrónicas intensas con las personas que integran la 
estructura escolar: estudiantes, administrativos, directivos y los otros académicos; los grupos que conforman esta estructura en muchos casos constituyen fuerzas más en pugna que en colaboración. Así, las dimensiones sincrónicas impactan simultáneamente en la constitución de la vocación docente y son determinantes en las prácticas del profesorado (Cárdenas y Santos, 2016).

Con respecto a la construcción de la vocación docente, los maestros no sólo la construyen en el transcurso del tiempo diacrónicamente, sino en el tiempo mismo, en los actos simultáneos del hacer relacionados con el entorno nacional, internacional e institucional, así como con las funciones específicas de su trabajo; elementos estructurales que se entrelazan con la vida docente e influyen en la construcción de su ser. En el trabajo cotidiano de la docencia existe gran cantidad de acciones que los maestros realizan: una de ellas y, tal vez, la principal es impartir cursos. Sin embargo, la enseñanza no es una acción libre de complejidades, sino que es una de las profesiones más complejas que existen, debido al abanico de responsabilidades que atienden y resuelven en los ámbitos académicos, educativos y administrativos. La pregunta clave es cómo los docentes asumen los elementos sincrónicos de la docencia en su cotidianidad. En este nivel no hay historia, sino simultaneidades que se entretejen en la vida docente cotidiana. En el eje estructural o sincrónico se pueden identificar tres grandes áreas que impactan la docencia: el entorno jurídico-político y axiológico-social, la tarea docente académica y educativa, y los componentes de la institución escolar. En cada una de estas áreas se mueven permanentemente construcciones y destrucciones que mueven al profesorado y sutilmente configuran estilos docentes, ambientes institucionales y dinámicas sociales que facilitan u obstruyen su tarea cotidiana, las cuales tienen consecuencias reales en la formación de los alumnos (Cárdenas y Santos, 2016).

Referente a los factores intrínsecos donde las subdimensiones: como debilidades a las que están sometidos, (desmotivación, estrés laboral), se indica un nivel elevado el que corresponde al $76 \%$ con la categoría siempre, seguido del $14 \%$ a veces y $10 \%$ nunca.

En la motivación existen factores extrínsecos (externos) e intrínsecos (internos) que definen el quehacer de cada persona. Es la vocación parte de la motivación intrínseca, sin desconocer que existen otros factores que modelan la motivación, como por ejemplo la sobrecarga de trabajo y estrés ocupacional, deterioro de las relaciones con otros 
docentes y directivos, déficits de formación y a los retos presentados por las nuevas tecnologías (González-Torres, 2003; Suárez, 2012, citado por Franco, Vélez, López y Becerra, 2016).

La motivación intrínseca, suele asociarse a las conductas y al rendimiento del individuo, permitiéndole alcanzar niveles mayores de eficacia en sus funciones (Gagné \& Deci, 2014; Ringelhan, Wollersheim, Welpe, Fiedler, \& Spörrle, 2013, Ibid). Así, las personas motivadas intrínsecamente reciben gratificaciones personales derivadas de sus propios intereses y no por recompensas provenientes del exterior como incentivos monetarios, ascensos o reconocimiento social (Gagné \& Deci, 2005; 2014, Ibid). En efecto, la motivación ha sido entendida como un factor determinante en el individuo para la escogencia consciente de una carrera específica como la de ser maestro (Albulescu \& Albulescu, 2015, Ibid).

Las actividades desafiantes y la retroalimentación positiva se relacionan directamente con las sensaciones de competencia y autonomía personales que influencian la satisfacción de las personas en su lugar de trabajo y en su vida. Justamente, un docente motivado es más propenso a desarrollar sus funciones con autonomía, implicación personal, iniciativa, perseverancia y compromiso de cambio hacia el mejoramiento de la calidad de la enseñanza (Lauretti, Villalobos, \& González, 2007, citado por Franco, Vélez, López y Becerra, 2016).

Respecto al clima laboral desarrollado, con la subdimensión: creando un clima desfavorable para alcanzar mayores niveles de eficacia en sus funciones, se índice un nivel medio que corresponde al $50 \%$ con la categoría siempre, seguido del $25 \%$ a veces y $25 \%$ nunca.

Es un hecho contrastado el papel crucial que juega el docente en la calidad y significatividad del aprendizaje de sus estudiantes (Marcelo, 2009). Situando la mirada en ese docente y cómo ha llegado a conformarse como tal, surge la necesidad de atender a su identidad profesional, entendiéndola como un proceso relacional, político y social (Rivas, Leite, Corté, Márquez y Padua, 2010) que tiene lugar en un espacio temporal extenso donde pueden identificarse momentos críticos para su construcción: las experiencias escolares, su percepción de la docencia, la formación inicial universitaria y, posteriormente como docente, el primer contacto con la profesión (Bolívar, 2006a; Bolívar, Domingo y Pérez, 2014, mencionado por Jarauta Borrasca, 2017). 
Junto a la adquisición de un conocimiento personal para la enseñanza, el conocimiento de uno mismo y el desarrollo de la identidad constituyen piezas claves en el proceso de convertirse en maestro (Connelly y Clandinin, 1990, mencionado por Jarauta Borrasca, 2017). La identidad, en su significado más amplio, ha sido un constructo exhaustivamente analizado desde varios ámbitos. La filosofía, la historia, la antropología, la psicología y la sociología, han abordado con diversos sentidos y bajo diferentes objetivos, la problemática de la construcción y desarrollo de la identidad.

\section{CONCLUSIÓN}

Se ha llegado a la conclusión de que la vocación docente se halla obstaculizada por factores extrínsecos, (remuneración, burocracias impuestas, la presión de los padres), como amenazas que pongan en riesgo la labor docente y por factores intrínsecos como debilidades a las que están sometidos, (desmotivación, estrés laboral) creando un clima desfavorable para alcanzar mayores niveles de eficacia en sus funciones.

La investigación tiene como objetivo analizar la vocación docente de la "Esc. Bás. $\mathrm{N}^{\mathrm{o}}$ 5639 Ntra del Pilar" y la "Esc. Bás. N 1.142 Santa María Magdalena” de la Ciudad de Pilar, año 2019-2020, a partir de las variables extrínsecas e intrínsecas, siendo que la rápida evolución de la sociedad moderna exige de la educación actual posturas que le lleven a encarar las situaciones que configuran los actores que inciden en ella; es decir, involucrar a docentes, padres y alumnos.

Desde un punto de vista organizativo, la educación tiene como objetivo transmitir conocimientos y creencias, esto permite captar el sistema educativo. Las condiciones laborales son retos inexorables para cualquier institución, pues existen evidencias suficientes que permiten afirmar que las buenas condiciones de trabajo son fundamentales para que todo trabajador rinda en forma a la hora de su desempeño. La actividad del docente en el aula es determinante para la enseñanza que éste desarrolla en frutos de aprendizaje en los alumnos. Y para que esto ocurra, los docentes están poniendo todos sus empeños de manera a propiciar las condiciones mínimas favorables a este agente tan decisorio en el proceso de enseñanza-aprendizaje. 


\section{REFERENCIA BIBLIOGRÁFICA}

Álvarez, (1995). Orientación Profesional. España: Editorial Cedecs.

Bernal, C. A. (2006). Metodología de la investigación: para administración, economía, humanidades y ciencias sociales. Segunda Edición. Editorial Pearson educación.

Cárdenas Olivares, G; y Santos Loyo, N. (2016). Sincronía y diacronía: una problematización de la vocación docente (segunda parte) Sincronía, núm. 70, julio-diciembre, 2016, pp. 244-266 Universidad de Guadalajara Guadalajara, México

Fernández, Y., González, N., Pompa V., Garcés, V., Figueredo, E., (2016). Motivación profesional en estudiantes de enfermería. Revista Multimed Médica Granma. ISSN 1028-4818 RPNS-1853.

Franco López, J; Vélez Salazar, F.M; López Arellano, H;. Becerra, M.A. (2016). Análisis de relevancia para la valoración de la vocación docente a partir de variables extrínsecas e intrínsecas: Caso colegios del área metropolitana de Medellín - Colombia

Gómez, G., Flores, J, Giménez, E. (1996). Metodología de la Investigación Cualitativa. Barcelona: Tauro.

González R., Cardentey, J. (2015). La orientación vocacional en residentes de Medicina General Integral. Revevista Arch Med Camagüey Vol19(6)2015.

Hernández Sampieri, R. (2005 ). Metodología de la Investigación. La Habana: Félix Varela.

Jarauta Borrasca, B. (2017). La construcción de la identidad profesional del maestro de primaria durante su formación inicial. El caso de la universidad de Barcelona Profesorado. Revista de Currículum y Formación de Profesorado, vol. 21, núm. 1, 2017, pp. 103-122 Universidad de Granada Granada, España.

Medina, C., Watanabe, R., Angulo C., (2018). Influencia de la vocación profesional en el rendimiento académico de los estudiantes de Medicina Veterinaria y Zootecnia de una universidad privada de Lima, Perú. Revista Inv Vet Perú 2018; 29(4): 1073-1086. Disponible en: http://dx.doi.org/10.15381/rivep.v29i4.15193.

Mendoza, I. Machado, E. Montes, N. (2016). La orientación vocacional y la elaboración de los proyectos personales de vida. Tendencias y enfoques la 
orientación vocacional y la elaboración de los proyectos personales de vida. Revista Cognosis; Filosofía, Letras y Ciencias de la Educación.

Mujica-Johnson, Felipe Nicolás; Orellana-Arduiz, Nelly del Carmen. (2019). Deseos vocacionales que incentivan a la formación docente en Educación Física Revista Educación, vol. 43, núm. 1, 2019 Universidad de Costa Rica, Costa Rica Disponible en: http://www.redalyc.org/articulo.oa?id=44057415041 DOI: https://doi.org/10.15517/revedu.v43i1.30013.

Rodríguez M., Trujillo P, Bolaños P., Leyva M. (2015). La orientación profesional: indicador de eficiencia en la formación de profesionales. Revista Edumecentro 7(4):178-95.

Disponible

en:

http://www.revedumecentro.sld.cu/index.php/edumc/article/view/449/pdf_1 